\title{
Evaluación estadística de los niveles de mercurio detectados en peces de pico, en Costa Rica, en el periodo 2003-2013
}

\section{Statistical Evaluation of the Levels of Mercury Detected in Billfish in Costa Rica, for 2003-2013}

Javier Rodríguez-Yáñez

urutico@gmail.com

Laboratorio de Ecología Urbana, Universidad Estatal a Distancia (UNED)

San José, Costa Rica

Gilberto Piedra-Marín

gilberto.piedra.marin@una.cr

Escuela de Química, Universidad Nacional (UNA)

Heredia, Costa Rica

Recibido-Received: 11/set/2015 / Aceptado-Accepted: 15/dic/2015 / Publicado-Published: 31/jul /2016.

\begin{abstract}
Resumen
Los peces de pico, como el marlín, el pez vela y el pez espada, son depredadores pelágicos superiores de la cadena alimenticia; los especímenes de gran tamaño contienen en sus tejidos niveles significativos de metilmercurio $\left(\mathrm{MeHg}^{+}\right)$. En el presente trabajo se revisaron, clasificaron, tabularon y analizaron estadísticamente todos los registros de análisis del mercurio, realizados en el periodo 2003-2013 por el Laboratorio Nacional de Servicios Veterinarios (LANASEVE), en pescados de pico. Los niveles de mercurio tuvieron un comportamiento de distribución tipo Poisson; la mayoría de los análisis se asociaron con muestras que oscilaban en el rango de $0,2 \mathrm{mg} \mathrm{Hg} / \mathrm{kg}$ a $0,4 \mathrm{mg} \mathrm{Hg} / \mathrm{kg}$ pescado. Se observó una mayor dispersión en los niveles de mercurio en marlín, con respecto al pez espada. La concentración promedio del mercurio en el marlín fue de 0,583 $\mathrm{mg}$ de $\mathrm{Hg} / \mathrm{kg}$ de pescado, en tanto para el pez espada fue de 0,533 $\mathrm{mg} \mathrm{de} \mathrm{Hg} / \mathrm{kg}$ de pescado. Los Valores de Ingesta Semanal Tolerable Recomendada (ISTR) para el consumo del marlín fueron $165 \mathrm{~g} / \mathrm{semana}$ para las mujeres embarazadas y $96 \mathrm{~g} / \mathrm{semana}$ para la niñez, en tanto para pez espada fueron $180 \mathrm{~g} / \mathrm{semana}$ y $105 \mathrm{~g} / \mathrm{semana}$, respectivamente. Los niveles de mercurio ligeramente mayores en marlín sugieren un menor consumo semanal comparado con el pez espada.
\end{abstract}

Palabras claves: Mercurio, análisis, estadística, peces de pico, Costa Rica, LANASEVE.

\begin{abstract}
The billfish, like marlin, fish sailing and swordfish are pelagic predators, top in the food chain; large specimens contain significant levels of methyl mercury $(\mathrm{MeHg}+)$ in their tissues. In the current work, all mercury analysis records in billfish were reviewed, classified, tabulated, and statistically analyzed, for the period 2003-2013 by Laboratorio Nacional de Servicios Veterinarios or LANASEVE (National Laboratory of Veterinary Services). The mercury levels had a Poisson type distribution behavior; the majority of the analysis were associated with samples fluctuating in the range of fish $0.2 \mathrm{mg} \mathrm{Hg} / \mathrm{kg}$ to $0.4 \mathrm{mg} \mathrm{Hg} / \mathrm{kg}$. Compared to swordfish, there was a greater dispersion in marlin mercury levels. Average mercury concentration in marlin was $0,583 \mathrm{mg} \mathrm{Hg} / \mathrm{kg}$, while in swordfish it was $0,533 \mathrm{mg} \mathrm{Hg} / \mathrm{kg}$. The Valores de Ingesta Semanal Tolerable Recomendada o ISTR (Tolerable Weekly Intake Values Recommended) for marlin consumption were $165 \mathrm{~g} /$ week for pregnant women and $96 \mathrm{~g} /$ week for children, meanwhile for swordfish were $180 \mathrm{~g} /$ week and $105 \mathrm{~g} /$ week, respectively. Slightly higher mercury levels in marlin suggest a lower weekly consumption compared to swordfish.
\end{abstract}

Keywords: Mercury, analysis, statistics, billffish, Costa Rica, LANASEVE. 
UNICIENCIA Vol. 30, No. 2, pp. 19-29. Julio-diciembre, 2016.

URL: www.revistas.una.ac.cr/uniciencia

Email: revistauniciencia@una.cr

Los peces de pico son depredadores pelágicos conocidos por su velocidad y su característica nariz en forma de pico. Poseen un cuerpo fusiforme, robusto, con fuertes aletas caudales que les permiten ser buenos nadadores y realizar migraciones de considerables distancias. Como parte de los peces pelágicos destacan los pertenecientes a la familia Istioforide, como el marlín, el pez vela y el pez espada; las zonas de mayor abundancia están situadas en la región de Florida y Venezuela en el Océano Atlántico; Perú y Baja California en el Océano Pacífico, y Australia en el Océano Índico-Pacífico (The Bilfish Foundation, 2015).

Recientemente, los peces de pico han sido considerados en los proceso de sobreexplotación, por lo que deben establecerse restricciones de consumo o venta (Departamento de pesca de la FAO, 1994). En algunos países se han establecido normas al respecto; por ejemplo, en los Estados Unidos de América se aprobó la Ley H.R. 2706, más conocida como Billfish Conservation Act (BCA), que prohíbe la venta de la mayoría de este tipo de peces (House of Representatives, 2012). En Costa Rica, la ley 8436 (Asamblea Legislativa de Costa Rica, 2005) declara en el artículo 76 al pez vela del Atlántico (Istiophorus albidius), al marlín rallado (Tetrapturas audaz), al marlín azul (Makaira nigrioans), al marlín negro (Makaira indica) y al sábalo (Melaops atlanticus) como especies de interés turístico-deportivo. Sin embargo, a raíz de la disminución considerable de las capturas del pez vela del Pacífico (Istiophorus platypterus), el Instituto Costarricense de Pesca (INCOPESCA) estableció restricciones a la exportación de dicha especie para prevenir el agotamiento de este recurso, con la consiguiente repercusión económica y social sobre todos los sectores de la sociedad que dependen directa o indirectamente de él (INCOPESCA, 2008).

Los peces de pico son depredadores superiores en la cadena alimenticia; los especímenes de gran tamaño, como los peces espada, los tiburones o ciertas especies de atún, contienen en sus tejidos niveles significativos del catión metilmercurio $\left(\mathrm{CH}_{3} \mathrm{Hg}^{+}\right)$, sustancia que previamente ingirieron sus presas. El mercurio es un metal que se presenta en tres formas químicas: mercurio elemental $\left(\mathrm{Hg}^{\circ}\right)$, mercurio inorgánico (como iones $\mathrm{Hg}_{2}{ }^{2+} \mathrm{y} / \mathrm{o} \mathrm{Hg}^{2+}$ ) y mercurio orgánico. Dentro de las diferentes formas del mercurio orgánico, el metilmercurio es la forma más común en la cadena alimenticia (EFSA, 2012), llega inclusive a representar más del $90 \%$ del Hg total (Agencia Catalana de Seguridad Alimentaria, 2011). El metilmercurio es muy tóxico para los riñones, el sistema cardiovascular y, sobre todo, para el sistema nervioso (Farré et al, 2010; Bloom, 1992) .

Los niveles del mercurio han sido evaluados en diferentes productos pesqueros, debido a su efecto tóxico sobre el ser humano; dicho efecto depende de la dosis consumida y de las características del sujeto expuesto, así como de la periodicidad de consumo. Con base en estas variables, se ha establecido el valor de Ingesta Semanal Tolerada Provisional (ISTP) para el metilmercurio en 1,6 mg Hg/kg de masa corporal (Joint FAO/WHO expert Committee on Food Additives, 2003).

La población de riesgo usualmente considerada para el ISTP la constituyen las mujeres embarazadas y la niñez con edades entre 7 y 12 años. La masa corporal promedio de una mujer embarazada oscila alrededor de los $60 \mathrm{~kg}$, en tanto los niños tienen una masa máxima de $35 \mathrm{~kg}$ (Farré et al, 2010). De acuerdo con lo anterior, la FAO/OMS propuso el valor de Ingesta Semanal Máxima Recomendada (ISTR) de los productos de la pesca, combinando los valores de ISTP con la masa corporal de la persona, la concentración del mercurio en el producto pesquero y la frecuencia de consumo (normalmente una semana), de acuerdo con la ecuación: 
ISSN Electrónico: 2215-3470

DOI: http://dx.doi.org/10.15359/ru.30-2.2
UNICIENCIA Vol. 30, No. 2, pp. 19-29. Julio-diciembre, 2016.

URL: www.revistas.una.ac.cr/uniciencia Email: revistauniciencia@una.cr

$\operatorname{ISTR}=\frac{[\operatorname{ISTP}(\mathrm{en} \mu \mathrm{g} / \mathrm{kg})] x[\text { masa corporal }(\mathrm{en} \mathrm{kg})] x 1000}{[\text { frecuencia del consumo }(\text { en semanas })] x[\text { concentración del Hg }(\mathrm{en} \mathrm{mg} / \mathrm{kg})] x 1000}$

El ISTR es expresado en gramos de pescado o marisco por semana. Los dos factores de 1000 surgen de la conversión de microgramos a miligramos y de kilogramos a gramos, respectivamente.

En Costa Rica, los niveles del mercurio en pescados son evaluados por el Laboratorio Nacional de Servicios Veterinarios (LANASEVE), el cual forma parte del Servicio de Sanidad Animal (SENASA) del Ministerio de Agricultura y Ganadería (MAG). Los criterios de aceptación de la muestra y los muestreos ejecutados por la Dirección de Inocuidad de Productos de Origen Animal (DIPOA) y la Dirección de Cuarentena Animal (DCA) del SENASA se basan en el Reglamento CE 333/2007 de la Unión Europea (Comisión de las Comunidades Europeas, 2007). El criterio establecido por DIPOA para el tamaño de la muestra de análisis se estableció en $1 \mathrm{~kg}$ por lote; paralelamente, consideró que la cantidad de las submuestras debía ser 10 para los lotes mayores a los $500 \mathrm{~kg}$. Si el lote a muestrear supera las 100 toneladas, se recomienda seccionarlo en sub-lotes de 15 toneladas, en tanto si es menor a 100 toneladas, se toma como un solo lote y se considera a granel (Comisión de las Comunidades Europeas, 2007; SENASA, 2012).

El método analítico que se utiliza para la determinación del mercurio en productos pesqueros realiza inicialmente una digestión química de la muestra, que transforma todo el mercurio presente a ion mercurio divalente $\left(\mathrm{Hg}^{2+}\right)$. Este último es, posteriormente, analizado en un equipo de absorción atómica (AA) con generación de hidruros. Los resultados del equipo de AA del LANASEVE son validados periódicamente, según las normas estipuladas en el Reglamento CE No 333/2007 (Comisión de las Comunidades Europeas, 2007).

En el presente trabajo se desarrolló un análisis estadístico descriptivo de los contenidos del mercurio en los pescados de pico (marlín, pez vela y pez espada) en el período 2003-2013, con la intención de establecer valores de ISTR de conformidad con las recomendaciones de consumo establecidos por la Organización Mundial de la Salud (OMS) y la Organización de las Naciones Unidas para la Agricultura y la Alimentación (FAO).

\section{Metodología}

Se revisaron, clasificaron y tabularon todos los registros del análisis de mercurio en pescados de pico realizados en el periodo 2003-2013 por el LANASEVE. La información se obtuvo a partir de los formularios de recepción de las muestras para los alimentos y de los reportes de análisis de mercurio total que genera el laboratorio de la unidad de Residuos y Contaminantes en Alimentos de Origen Acuático (RECAA) del Departamento Inocuidad de Alimentos (DIA) del LANASEVE.

Se realizaron un total de 2503 análisis de mercurio en pescados y en productos pesqueros durante el 2003 al 2013, de los cuales 370 correspondieron al marlín y 296 al pez espada (los análisis de estos pescados corresponden al $29 \%$ del total de los análisis realizados en el periodo). El pez vela tiene muy poca cantidad de análisis reportados, por lo que no se considerará en este estudio. En algunos casos existe información incompleta en el formulario de recolección de las muestra, sobre todo con respecto a los tamaños del lote y al destino (importación, exportación, consumo local, etc.), lo que no permite su evaluación estadística (LANASEVE, 2012). En consecuencia, los datos utilizados en el análisis estadístico se restringieron a los registros que: 
UNICIENCIA Vol. 30, No. 2, pp. 19-29. Julio-diciembre, 2016.

a) tenían la información completa, b) no incluían productos enlatados o a granel, y c) cuyos tamaños de lote se encontraban en el rango $500 \mathrm{~kg}$ a $100000 \mathrm{~kg}$. De acuerdo con estos criterios, se tienen 341 registros útiles para el marlín (del total de 370 registros) y 277 (de los 296 registros) para el pez espada.

\section{Resultados y discusión}

La Unidad Residuos y Contaminantes en Alimentos de Origen Acuático (RECAA) del Departamento Inocuidad de Alimentos (DIA) del LANASEVE realizó los muestreos del pescado para los respectivos análisis de inocuidad. Para la recolección de la información se utilizaron los formularios de recepción de muestras para alimentos y los reportes de análisis de mercurio total que genera el LANASEVE. A partir de estos documentos se obtuvo la información asociada al producto pesquero, al tamaño del lote, al destino y al contenido del mercurio. En estos formularios no se anotaba el nombre científico de la especie en cuestión sino, más bien, el nombre común o comercial; en consecuencia, no se hacía una diferenciación por especie. En la tabla 1 se enumeran las diferentes especies de pescados de pico consideradas en el presente trabajo.

Tabla 1

Nombres científicos de las especies asociadas a los nombres comunes del marlín, el pez espada y el pez vela

\begin{tabular}{cl}
\hline Nombre común & Nombre científico de la(s) especie(s) incluidas \\
\hline Pez espada & Xiphias gladius \\
Pez vela & Istiophorus albicans \\
& Istiophorus platypterus \\
Marlín & Makaira indica \\
& Makaira mazara \\
& Tetrapturus audax \\
\hline
\end{tabular}

Nota: (Mar Viva, 2010).

El ordenamiento por lotes provocó algunos inconvenientes a la hora de sistematizar la información y realizar el análisis estadístico; por ejemplo, es posible establecer más de un destino: además de las categorías básicas de consumo nacional ( $\mathrm{Nac}$ ), importación (Imp) y exportación (Exp), se pueden realizar combinaciones entre ellas para generar otras; se observó que en general el destino nacional se combinaba con exportación (Nac/Exp) e importación (Imp/Nac). Adicionalmente, se consideraban lotes de vigilancia (Vig) asociados al control sanitario o aleatorio.

En la figura 1 se muestra la distribución de los valores de la concentración, en mg de Hg/ $\mathrm{kg}$ de pescado para el marlín (Figura 1a) y el pez espada (Figura 1b), en función del tamaño del lote $(\mathrm{kg})$. Debido a la variación tan amplia en los tamaños de lote se decidió emplear escala logarítmica. Se observó que, con respecto al marlín, el 85 \% de los análisis del mercurio realizados corresponden a lotes cuyos tamaños oscilaban entre los $500 \mathrm{~kg}$ y los $20000 \mathrm{~kg}$; en tanto para el 
pez espada el $56 \%$ de los análisis se asociaban con lotes en el rango de $500 \mathrm{~kg}$ a $5000 \mathrm{~kg}$. Con base en los criterios establecidos previamente, se determinó que el $92 \%$ de los datos del marlín se ubicaba dentro del rango de interés estadístico, en tanto para pez espada fue del $94 \%$.
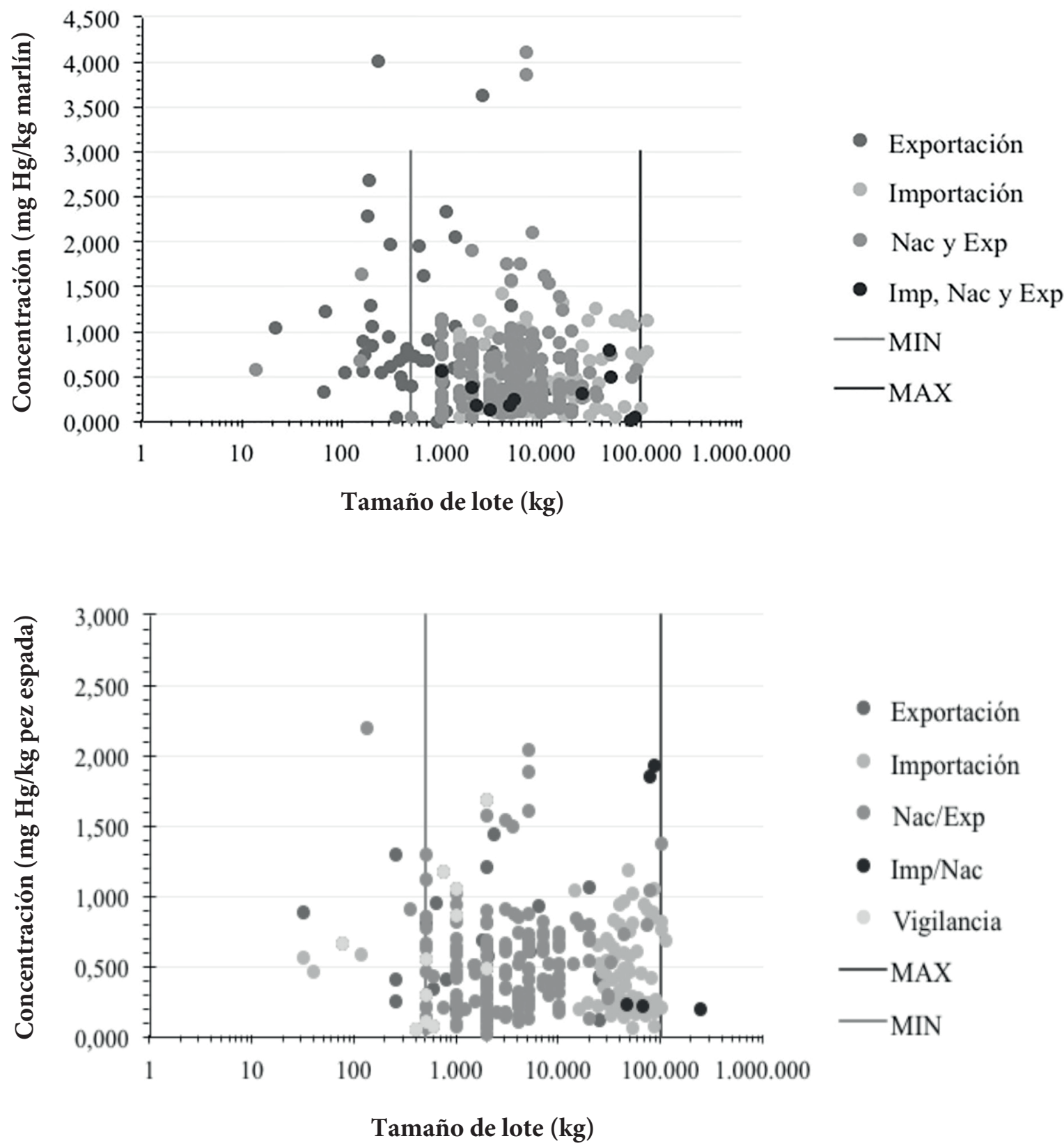

Figura $1 \boldsymbol{a} y \boldsymbol{b}$. Distribución de los valores de concentración en (mg de $\mathrm{Hg} / \mathrm{kg}$ de pescado), para el marlín y el pez espada, con respecto al tamaño del lote $(\mathrm{kg})$. Datos propios de la investigación. 
La figura 2 muestra la distribución de los análisis del mercurio en el marlín y el pez espada en función del destino. Se observa que la mayoría de los análisis del mercurio en ambos pescados se asociaron con el destino Nac/Exp y en un menor grado con Imp. Los análisis asociados a la vigilancia (Vig) solamente se realizaron con el pez espada.

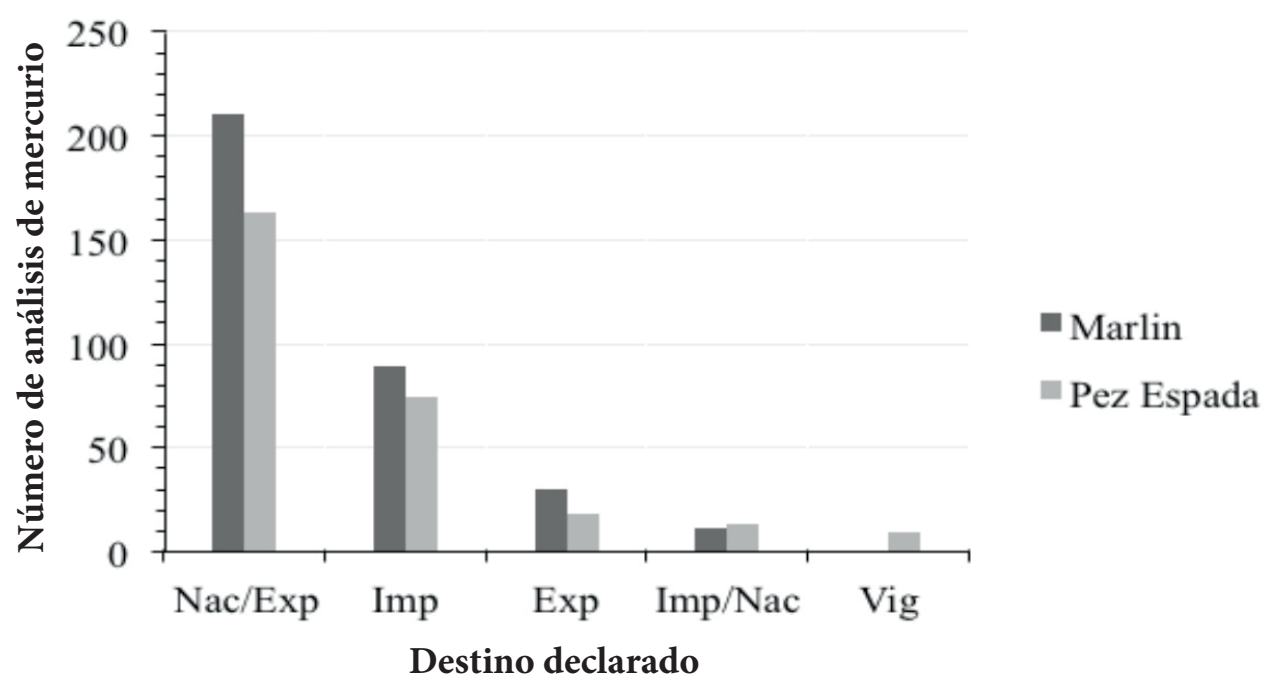

Figura 2. Cantidad de análisis por destino de pescado, para el marlín y el pez espada en el rango de $500 \mathrm{~kg}$ a $10000 \mathrm{~kg}$. Datos propios de la investigación.

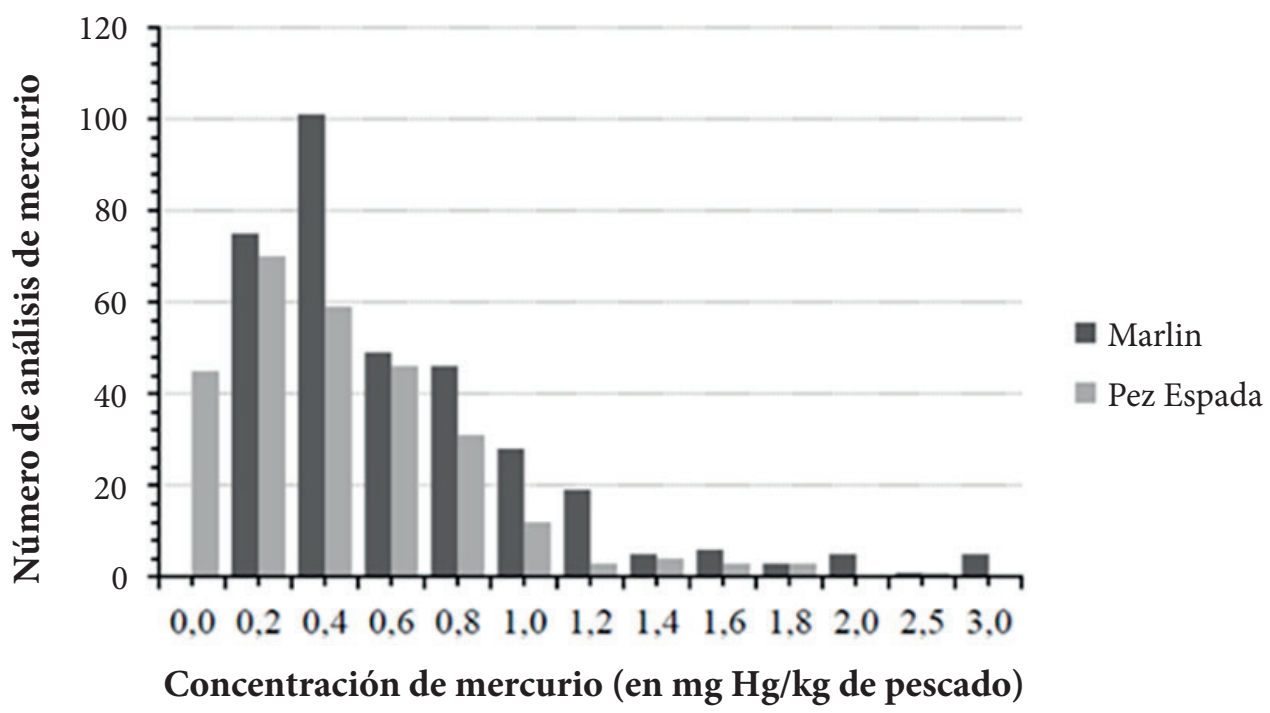

Figura 3. Cantidad de análisis por rango de concentración en (mg de $\mathrm{Hg} / \mathrm{kg}$ de pescado), para el marlín y el pez espada. Datos propios de la investigación. 
ISSN Electrónico: 2215-3470 DOI: http://dx.doi.org/10.15359/ru.30-2.2
UNICIENCIA Vol. 30, No. 2, pp. 19-29. Julio-diciembre, 2016. URL: www.revistas.una.ac.cr/uniciencia Email: revistauniciencia@una.cr

La distribución de los análisis del mercurio en los pescados de pico, en función de los niveles encontrados de $\mathrm{Hg}$, se muestra en la figura 3. Se observa que la mayoría de los análisis se asociaron con muestras de pescado cuyos rangos de concentración oscilaban entre 0,2 mg $\mathrm{Hg} / \mathrm{kg}$ pescado y $0,4 \mathrm{mg} \mathrm{Hg} / \mathrm{kg}$ pescado. Adicionalmente, la mayoría de los análisis mostraron concentraciones de mercurio por debajo del valor límite recomendado para los pescados depredadores ( $1 \mathrm{mg} \mathrm{Hg} / \mathrm{kg}$ ); de hecho, solo el $12 \%$ de los análisis de mercurio en el marlín sobrepasó el valor recomendado, en tanto el $5 \%$ de los análisis en pez espada superó el valor de referencia.

En la figura 4 a y b se muestran los diagramas de caja y bigote para la distribución de las concentraciones del mercurio en el marlín y en el pez espada. Este tipo de gráficos se utiliza para evaluar la distribución de los datos en torno a un valor de tendencia central, en este caso la mediana, así como la dispersión del conjunto de datos; la caja muestra la concentración de los datos alrededor de la mediana y su dispersión (simétrica o asimétrica) de los cuartiles con respecto a ella. Se observa que los rangos de las concentraciones del mercurio en el marlín son mayores que en el pez vela (salvo en el destino de Imp/Nac en la cual se observa el comportamiento opuesto); adicionalmente, el recorrido intercuartílico es mayor en el marlín, lo que sugiere una mayor dispersión en los niveles del mercurio en este pescado. La ausencia de la información relacionada con el tamaño (masa), la edad y la zona de captura, entre otros, no permite explicar la mayor dispersión en los niveles del mercurio encontrados en el marlín. Ambas distribuciones son asimétricas positivas (hacia la derecha o hacia valores mayores) y la mayoría de los datos tienden a concentrarse hacia la parte inferior de la distribución. El comportamiento de las medianas y los cuartiles indica que existen diferencias en las distribuciones de datos según el destino declarado. Los análisis del mercurio asociados con el destino Nac/Imp se comportaron de forma opuesta al comportamiento observado en los otros destinos.

En la tabla 2 se resumen los valores de los estadísticos asociados a estas distribuciones de datos. La distribución de los datos es de tipo Poisson; en este tipo de sistemas la media y la mediana generalmente no coinciden, sino que están ligeramente desfasadas, usualmente la mediana se ubica hacia valores menores, como ocurre en este caso. La concentración promedio del mercurio en el marlín en el periodo de estudio fue de 0,583 mg de $\mathrm{Hg} / \mathrm{kg}$ de pescado, con una desviación de $0,546 \mathrm{mg}$ de $\mathrm{Hg} / \mathrm{kg}$ de pescado; que al incorporarlo en la ecuación 1 arroja un ISTR de $165 \mathrm{~g}$ marlín/semana para las mujeres embarazadas y $96 \mathrm{~g}$ marlín/semana para los niños. En lo que respecta al pez espada, el nivel promedio del mercurio fue 0,533 $\mathrm{mg} \mathrm{de} \mathrm{Hg} /$ $\mathrm{kg}$ de pescado con una desviación de 0,354 mg de $\mathrm{Hg} / \mathrm{kg}$ de pescado; lo anterior resulta en valores de ISTR de 180 g y 105 g pez espada/semana para las mujeres embarazadas y niños, respectivamente. Tales resultados se resumen en la tabla 3. Se han calculado valores de ISTR para otros pescados (ELIKA, 2005; Farré, 2010; FDA, 2014). 
UNICIENCIA Vol. 30, No. 2, pp. 19-29. Julio-diciembre, 2016.

ISSN Electrónico: 2215-3470

URL: www.revistas.una.ac.cr/uniciencia

Email: revistauniciencia@una.cr

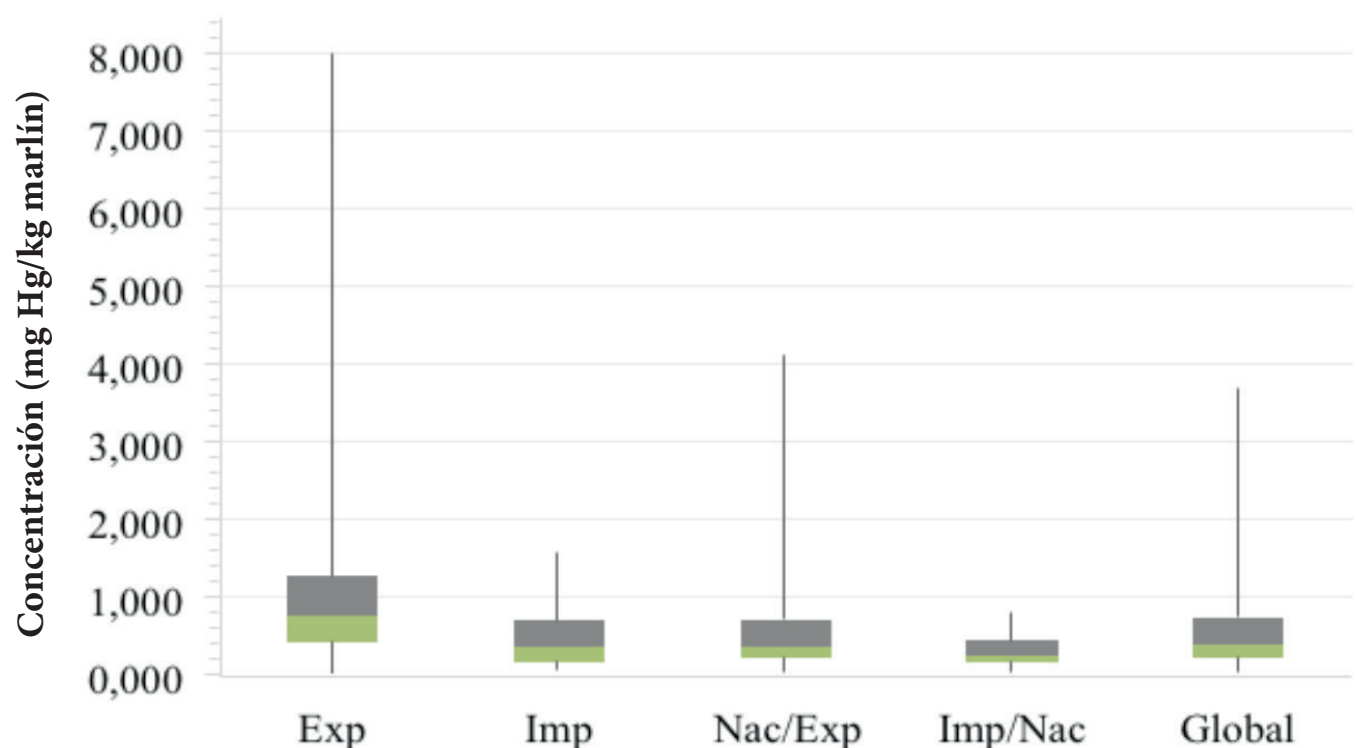

Destino declarado

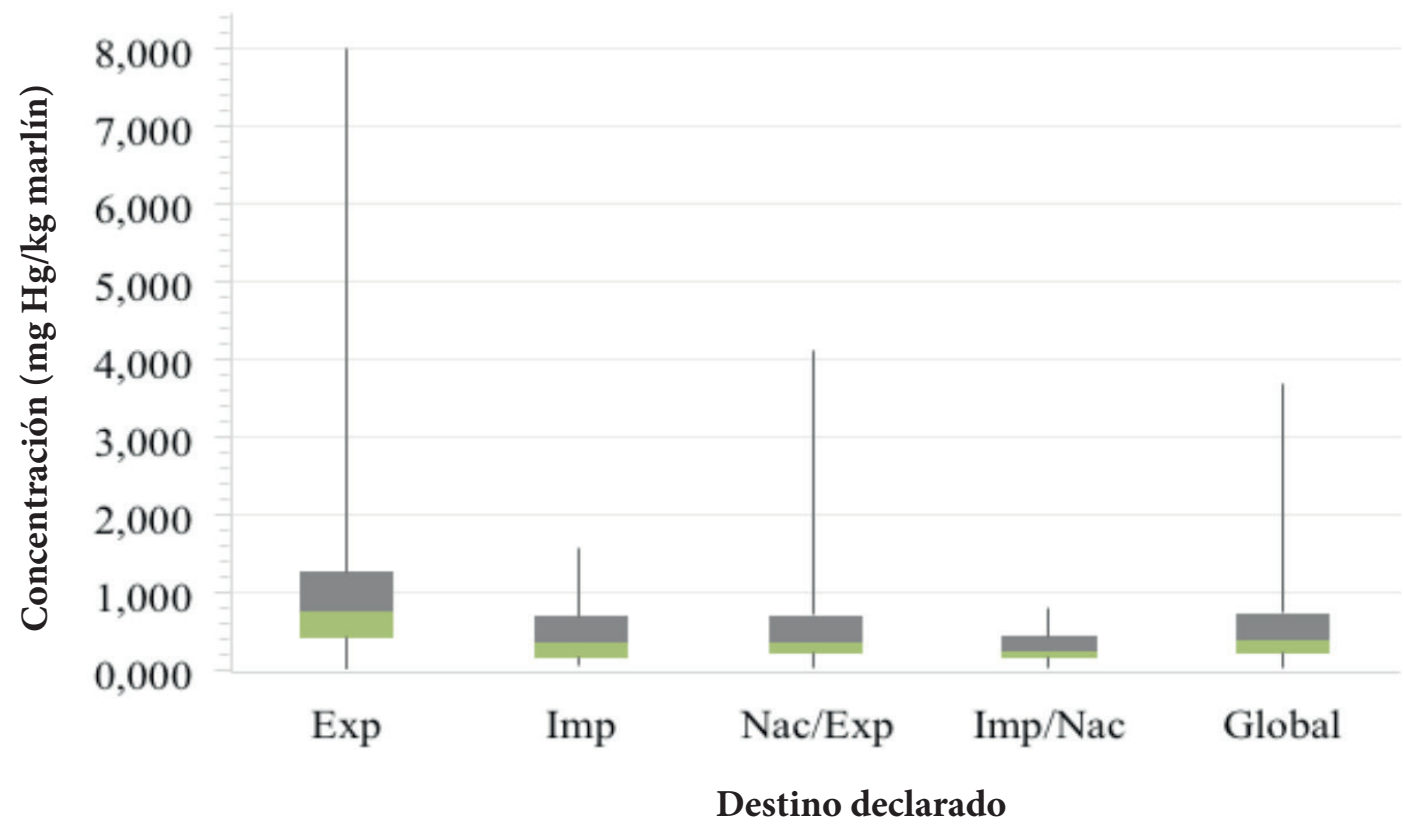

Figura 4 a y b. Diagramas de caja y bigote para el marlín y el pez espada, según el destino declarado, en el rango de $500 \mathrm{~kg}$ a $10000 \mathrm{~kg}$. Datos propios de la investigación. 
ISSN Electrónico: 2215-3470

DOI: http://dx.doi.org/10.15359/ru.30-2.2
UNICIENCIA Vol. 30, No. 2, pp. 19-29. Julio-diciembre, 2016. URL: www.revistas.una.ac.cr/uniciencia Email: revistauniciencia@una.cr

Tabla 2

Valores obtenidos de los estadísticos: Promedio, desviación estándar, mediana, Q25, y Q75 para la concentración en $\mathrm{mg}$ de $\mathrm{Hg} / \mathrm{kg}$ de pescado para el marlín y el pez espada

\begin{tabular}{lcccccccccccccc}
\hline \multirow{2}{*}{ Estadístico } & \multicolumn{1}{c}{ Marlín } & Exp & Imp & Nac/Exp & Imp/Nac & Vig & Global & Exp & Imp & Nac/Exp & Imp/Nac & Vig & Global \\
\hline Promedio & 1,383 & 0,500 & 0,519 & 0,307 & dnd & $\mathbf{0 , 5 8 3}$ & 0,630 & 0,461 & 0,524 & 0,813 & 0,703 & $\mathbf{0 , 5 3 3}$ \\
Desviación & 1,467 & 0,386 & 0,498 & 0,238 & dnd & $\mathbf{0 , 5 4 6}$ & 0,376 & 0,283 & 0,348 & 0,674 & 0,540 & $\mathbf{0 , 3 5 4}$ \\
\hline Mínimo & 0,004 & 0,056 & 0,048 & 0,018 & dnd & $\mathbf{0 , 0 4 5}$ & 0,124 & 0,071 & 0,021 & 0,080 & 0,080 & $\mathbf{0 , 0 4 6}$ \\
Q 25 & 0,436 & 0,160 & 0,238 & 0,154 & dnd & $\mathbf{0 , 2 3 2}$ & 0,356 & 0,231 & 0,263 & 0,237 & 0,300 & $\mathbf{0 , 2 6 1}$ \\
Mediana & 0,755 & 0,368 & 0,368 & 0,251 & dnd & $\mathbf{0 , 3 9 8}$ & 0,592 & 0,411 & 0,476 & 0,550 & 0,550 & $\mathbf{0 , 4 7 2}$ \\
Q 75 & 1,290 & 0,698 & 0,707 & 0,443 & dnd & $\mathbf{0 , 7 4 7}$ & 0,899 & 0,645 & 0,671 & 1,180 & 1,056 & $\mathbf{0 , 7 1 5}$ \\
Máximo & 8,026 & 1,566 & 4,110 & 0,796 & dnd & $\mathbf{3 , 6 8 4}$ & 1,440 & 1,191 & 2,040 & 1,933 & 1,692 & $\mathbf{1 , 7 5 9}$ \\
\hline
\end{tabular}

Nota: El promedio y la desviación (desv.) están calculados con base en regresiones normales. El término dnd se refiere a dato no disponible. Datos propios de la investigación.

Tabla 3

Valores de la ingesta semanal tolerable recomendada (ISTR), en g pescado/semana, para pescados de pico en grupos de riesgo

\begin{tabular}{lcccc}
\hline \multirow{2}{*}{ Pescado } & $\begin{array}{c}\text { Concentración promedio } \\
\text { (en } \mathbf{~ m g ~ H g / k g ~ p e s c a d o ) ~}\end{array}$ & & \multicolumn{2}{c}{ ISTR (g pescado/semana) } \\
& 0,583 & Mujeres embarazadas & Niños $^{*}$ \\
\hline Marlín & 0,533 & 165 & 96 \\
Pez espada & 180 & 105 \\
\hline
\end{tabular}

Nota: ${ }^{*}$ Niños con edades en el rango de 7 a 12 años. Datos propios de la investigación.

\section{Conclusión}

Se analizaron estadísticamente los niveles de mercurio en marlín y pez espada en los estudios realizados por el LANASEVE durante el periodo 2003 a 2013. Los destinos principales de ambos pescados fueron Nac/Exp y, en menor grado, Imp. La mayoría de los análisis mostraron concentraciones del mercurio por debajo del valor límite de la normativa de $1 \mathrm{mg} \mathrm{Hg} / \mathrm{kg}$ pescado para pescados depredadores (solo el $12 \%$ de los análisis del mercurio en el marlín sobrepasó el valor recomendado, en tanto en pez espada el $5 \%$ de los análisis superó el valor de referencia). Se observó una mayor dispersión en los niveles del mercurio en el marlín en comparación con el pez espada. Se obtuvieron valores de ISTR para el marlín y el pez espada a partir de la concentración promedio del mercurio de cada pescado: $160 \mathrm{~g}$ marlín/semana y $180 \mathrm{~g}$ pez espada/semana para mujeres embarazadas; $96 \mathrm{~g}$ marlín/semana y 105 g pez espada/semana para niños en el rango de edades de 7 a 12 años. Los niveles del mercurio ligeramente mayores en el marlín sugieren un menor consumo semanal de este tipo de pescado en la población de riesgo, tanto en las mujeres embarazadas como en los niños cuyas edades se encuentren en el rango de 7 a 12 años. En ese sentido, se hace necesario que el consumidor esté consciente de lo que ingiere y que tome las medidas pertinentes para proteger su salud. 
UNICIENCIA Vol. 30, No. 2, pp. 19-29. Julio-diciembre, 2016.

URL: www.revistas.una.ac.cr/uniciencia

Email: revistauniciencia@una.cr

\section{Agradecimientos}

Los autores agradecen a SENASA el aporte de los datos básicos y la información asociada a dichos análisis.

\section{Referencias}

Agencia Catalana de Seguridad Alimentaria. Metilmercurio. (2011). ACSA Brief Salud y Seguridad Alimentaria, Departament de Salut 2011. Recuperado de http://www.gencat.cat/salut/acsa/html/es/.../ acsabrief08-2011metilmercurio.pdf

Asamblea Legislativa de la República de Costa Rica. (2005). Ley 8436. Ley de Pesca y Acuicultura. Publicado en La Gaceta, número 78, del 25 de abril del 2005.

Bloom, N. S. (1992). On the methylmercury content of fish tissue. Canadian Journal of Fisheries and Aquatic Sciences. 49, 92113.

Comisión de las Comunidades Europeas. (2007). Reglamento (CE) No 333/2007 de la Comisión de 28 de marzo de 2007 por el que se establecen los métodos de inorgánico, 3-MCPD y benzo(a)pireno en los productos alimenticios. Diario Oficial de la Unión Europea 2007, 88/29-88/38. Recuperado de http:// www.boe.es/doue/2007/088/L00029-00038.pdf.

Departamento de Pesca de la FAO. (1994). Examen de la situación mundial de las especies altamente migratorias y las poblaciones transzonales. FAO Documento Técnico de Pesca. No. 337. Roma, FAO. 75p. ISBN 92-5-303523-4. Recuperado de: http:/ http://www.fao.org/docrep/003/t3740s/t3740s00.htm

EFSA. (2012). Scientific Opinion on the risk for the public health related to the presence of mercury and methyl mercury in food. EFSA Journal, 10(12), 2985.

ELIKA. (2005). Mercurio en pescado. Araba, España: Fundacion Vasca para la Seguridad Agroalimentaria.

Farré. R., Cameán. A. M., Vidal, M. C., Santacruz, A. L., Teruel, V. J. y Canales, E. T. (2010). Informe del Comité Científico de la Agencia Española de Seguridad Alimentaria y Nutrición (AESAN) en relación a los niveles de mercurio establecidos para los productos de la pesca. Revista del Comité Científico, 12, 29-36. Recuperado de http://aesan.msssi.gob.es/AESAN/docs/docs/evaluacion_riesgos/comite cientifico/MERCURIO P.PESCA.pdf

FDA. (2014). Mercury Levels in Commercial Fish and Shellfish (1990-2010). Silver Spring, U.S.A.: U.S. Food and Drug Administration. Recuperado de: http://www.fda.gov/food/foodborneillnesscontaminants/metals/ucm115644.htm

House of Representatives. (2012). Billfish Conservation Act of 2012. Recuperado de www.congress. gov/112/crpt/hrpt656/CRPT-112hrpt656.pdf

INCOPESCA (2008). Comunicación de acuerdo 476-2008. Recuperado de http://www.fecop.org/wpcontent/.../ACUERDO-476-2008-PEZ-VELA-1.pdf

Joint FAO/WHO Expert Committee on Food Additives. (2003). Contaminants: Methylmercury. En WHO Technical Report Series, 922 (Ed.). Evaluation of Certain Food Additives and Contaminants: Sixty-first report of the Joint FAO/WHO Expert Committee on Food Additives 2003; 132-141. Rome, Italy. Recuperado de http://whqlibdoc.who.int/trs/WHO_TRS_922.pdf

LANASEVE. (2012). Formulario SEG-PE-001-RE-002 Recepción de muestras para alimentos (xls). Recuperado de: http://www.senasa.go.cr/senasa/sitio/index.php/archivos/view/1098.

Mar Viva, INCOPESCA, MEIC. (2012). Guía de identificación de filetes de pescado y mariscos. Recuperado de http://marviva.net/images/Consumo Responsable/guia de identificacion de filetes de pescado_y mariscos.pdf 
ISSN Electrónico: 2215-3470

DOI: http://dx.doi.org/10.15359/ru.30-2.2
UNICIENCIA Vol. 30, No. 2, pp. 19-29. Julio-diciembre, 2016.

URL: www.revistas.una.ac.cr/uniciencia Email: revistauniciencia@una.cr

SENASA. (2012). Toma y manejo de muestras oficiales en productos pesqueros. Dirección de Inocuidad de Productos de Origen Animal. Recuperado de: http://www.senasa.go.cr/senasa/sitio/files/120912023910.pdf

The Billfish Foundation. (2015). What are billfish? Recuperado de http://www.billfish.org/education/ what-are-billfish/

\section{(2) $\odot \Theta \Theta$}

Evaluación estadística de los niveles de mercurio detectados en peces de pico, en Costa Rica, en el periodo 2003-2013 (Javier Rodríguez-Yáñez y Gilberto Piedra-Marín) por Revista Uniciencia se encuentra bajo una Licencia CreativeCommons Atribución-NoComercial-SinDerivadas 3.0 Unported. 